\title{
Disruption of Mycobacterium smegmatis Biofilms Using Bacteriophages Alone or in Combination with Mechanical Stress
}

\author{
Brendan Kiefer, John L. Dahl* \\ Department of Biology, University of Minnesota Duluth, Duluth, MN, USA \\ Email: ${ }^{*}$ jldahl@d.umn.edu
}

Received 29 July 2015; accepted 22 September 2015; published 25 September 2015

Copyright (C) 2015 by authors and Scientific Research Publishing Inc.

This work is licensed under the Creative Commons Attribution International License (CC BY). http://creativecommons.org/licenses/by/4.0/

C) (†) Open Access

\begin{abstract}
Environmental mycobacteria are capable of forming biofilms in low-nutrient environments, and these biofilms may act as reservoirs for opportunistic infections. The purpose of this study was to determine if bacteriophages could disrupt existing biofilms of acid-fast staining Mycobacterium smegmatis. Using the MBEC 96-well plastic peg assay system, M. smegmatis biofilms were created and then tested for their stability in the presence of mycobacteriophages isolated from a Minnesota sphagnum peat bog. All phages tested were lytic and were observed to have weak, intermediate, and strong abilities to disrupt $M$. smegmatis biofilms. The formation of biofilms was severely impaired in the presence of mycobacteriophages. Phage treatment was also shown to augment $M$. smegmatis biofilm disruption by mechanical forces of sonication or water flow. Our study shows that, as with biofilms of Gram-positive and Gram-negative bacteria, mycobacterial biofilms are also susceptible to destruction by bacteriophages.
\end{abstract}

\section{Keywords}

Mycobacterium smegmatis, Biofilm, Mycobacteriophage, Bacteriophage

\section{Introduction}

The genus Mycobacterium contains over 120 species, including saprophytic and pathogenic bacteria [1]. Mycobacteria can be classified into two groups: obligate pathogens such as Mycobacterium tuberculosis and Mycobacterium leprae, and environmental mycobacteria (EM), such as Mycobacterium avium complex. While the

"Corresponding author.

How to cite this paper: Kiefer, B. and Dahl, J.L. (2015) Disruption of Mycobacterium smegmatis Biofilms Using Bacteriophages Alone or in Combination with Mechanical Stress. Advances in Microbiology, 5, 699-710.

http://dx.doi.org/10.4236/aim.2015.510073 
majority of human infections are attributed to the tuberculosis complex of mycobacteria, EM are increasingly relevant in clinical settings as the cause of opportunistic infections that include skin lesions, pulmonary infections, lymphadenitis in children, endocarditis, meningitis, and disseminated disease [2]-[4]. EM infections are expected to increase due to expanding groups of the elderly, HIV-infected individuals, and those on immunosuppressive therapy [5]. EM are transmitted from aquatic and environmental sources primarily by ingestion, inhalation, and inoculation [6].

Bacteria typically inhabit their environments by forming biofilms, surface-attached communities of bacteria that are embedded in an extracellular polymeric substance (EPS) [7]. The composition of EPS varies based on the bacterial species and can contain polysaccharides, proteins, nucleic acids, and lipids [8]-[11]. Numerous mycobacteria, including Mycobacterium smegmatis, have been observed to grow in biofilms [12]-[16]. However, it is unclear if biofilm formation plays a role in pathogenic mycobacteria like M. bovis [17], M. mariunum [18], and M. lulcerans [19]. EM are highly enriched in showerheads, and a source of Mycobacterium avium has been traced to home showers of those infected [20]-[22]. Biofilm-borne bacteria exhibit a unique physiology compared to their planktonic (free-floating) counterparts. Biofilm cells may have increased resistance to environmental stresses like low pH, UV exposure, salinity, and dehydration [23]-[25]. Physiological heterogeneity and reduced growth rates inside biofilms confer resistance to disinfectants and antibiotics [12] [13] [26] [27]. The ability to adhere to surfaces combined with a high resistance to chlorine-based disinfectants enables mycobacteria to colonize municipal water systems [28]-[31]. This highlights the need for alternative biofilm intervention strategies for EM.

Since the discovery of bacteriophages over 100 years ago, the ability of phages to sanitize surfaces and treat human diseases has been examined. More recently, the ability of bacteriophages to reduce biofilm populations was demonstrated in a number of clinically relevant species: Escherichia coli, Pseudomonas aeruginosa, Staphylococcus epidermidis, Pseudomonas fluorescens, and Staphylococcus aureus [26] [32]-[36]. No previous studies have examined the interaction of bacteriophages in mycobacterial biofilms. Here we show that environmental mycobacteriophages can disperse existing M. smegmatis biofilms and can prevent these biofilms from forming initially.

\section{Materials and Methods}

\subsection{Bacterial Strains and Growth Condition}

Biofilms were generated using Mycobacterium smegmatis $\mathrm{mc}^{2}$ 155. Cells were grown on 7H11 agar (Difco) for $3-4$ days at $37^{\circ} \mathrm{C}$ before inoculating into $7 \mathrm{H} 9$ broth supplemented with $0.05 \%(\mathrm{v} / \mathrm{v})$ Tween 80 and growing to logarithmic phase $\left(\mathrm{OD}_{600} 0.75\right)$ at $37^{\circ} \mathrm{C}$ prior to use in biofilm assays.

\subsection{MBEC Biofilm Assay}

Log-phase cultures of $M$. smegmatis were pelleted and suspended in 7H9 broth without Tween 80 before bacteria were allowed to attach to plastic pegs of MBEC assay plates (MBECTM Biofilm Technologies Ltd., Calgary, Alberta). MBEC plates were gently rocked at room temperature (RT) with fresh media changes every 3 days. Biofilms were quantified using two different assays: 1) crystal violet (CV) staining of attached biofilms, and 2) serial dilutions to count colony-forming units (CFUs) following biofilm disruption. Quantification of M. smegmatis biofilms by CV staining closely approximates quantification by determining viable cell counts in a biofilm (Figure 1). Serial dilution plate counting was performed by aseptically removing pegs containing biofilms from MBEC plates with sterile pliers and placing them in individual microcentrifuge tubes containing 200- $\mu$ l aliquots of 7H9broth without Tween 80 . The tubes were gently vortexed to remove unbound cells and the fluid was discarded. Remaining biofilm cells were recovered from the pegs by adding them to 200 - $\mu$ l aliquots of 7H9 broth with Tween80 and sonicating them in a water bath sonicator (FS20, Fisher Scientific) for 5 min. CFUs were enumerated by serial dilutions and plate counting on 7H11 agar plates.

CV staining was performed, as previously described [37]. Briefly, MBEC plates containing biofilms were rinsed in phosphate buffered saline (PBS) to remove planktonic cells before fixing biofilms in methanol for 15 min and allowing them to dry. The plates were then stainined in a CV solution $(0.5 \%$, w/v) for 30 min before rinsing with PBS. Stained MBEC plates were placed into a 96-wellmicrotitre plate wells containing $150 \mu \mathrm{l}$ of $33 \%$ 


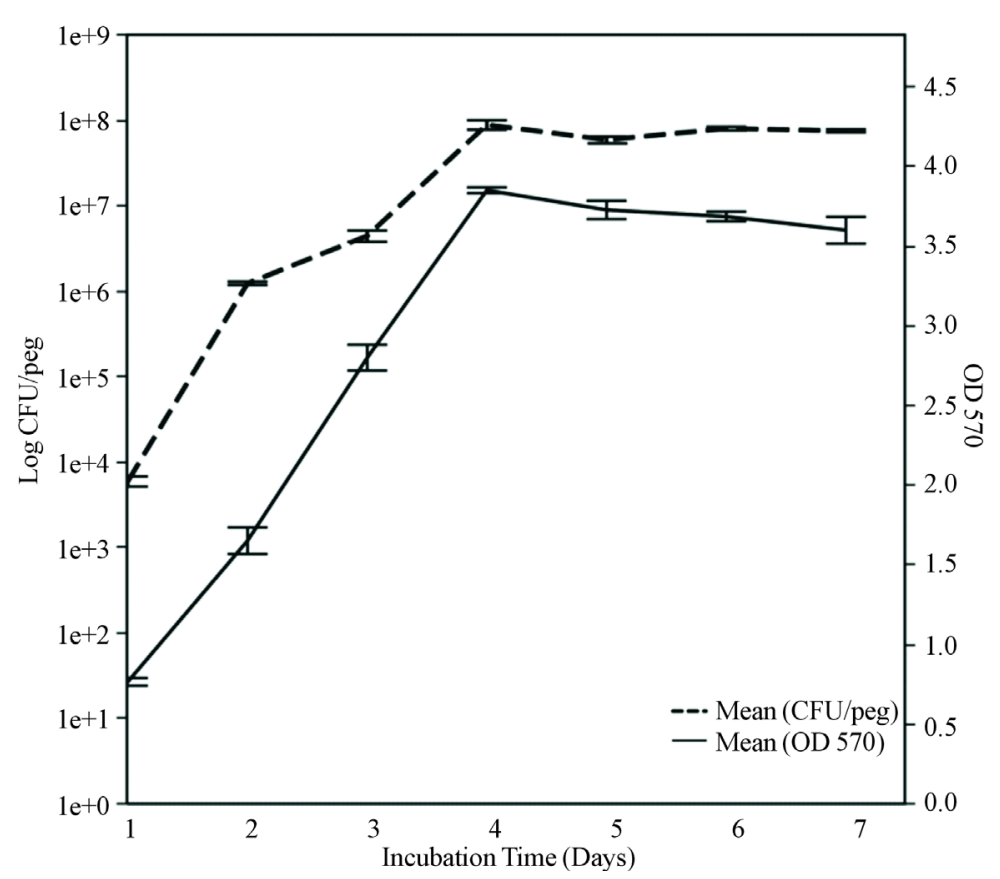

Figure 1. Growth of $M$. smegmatis biofilm in 7H9 broth in the MBEC device. Bacterial counts were performed by removing three pegs and extracting bacteria by water bath sonication. Viable counts were determined by serial dilution. Crystal violet (CV) staining was performed and the retained stain was quantified. The solid line represents the mean CFU/peg of three independent experiments $(n=9)$. The bars represent standard error (SE) and the dashed line represents the mean absorbance of dye extracted from pegs of three independent experiments $(n=9)$.

(v/v) glacial acetic acid for $20 \mathrm{~min}$. Eluted stain was quantified with a microtitre plate reader at $570 \mathrm{~nm}$ (SpectraMax M3, Molecular Devices).

\subsection{Isolation and Production of Phages}

Liquid samples were collected from the aqueous layers of a peat bog in the Big Bog State Recreation Area (Waskish, MN). These environmental samples were first enriched for phages by adding $M$. smegmatis and incubating at RT for 7 days. Samples were then centrifuged at $3000 \times \mathrm{g}$ for $10 \mathrm{~min}$ at RT, and the supernatants were passed through $0.2-\mu \mathrm{m}$ filters. Individual phages were isolated using an agar overlay technique. Briefly, filtered bog supernatants were incubated with log-phase $M$. smegmatis overnight in phages buffer (10 mM Tris, $\mathrm{pH} 7.5$, $10 \mathrm{mM} \mathrm{MgSO}_{4}, 68 \mathrm{mM} \mathrm{NaCl}$, and $1 \mathrm{mM} \mathrm{CaCl}_{2}$ ). These mixtures were then suspended in $5 \mathrm{ml}$ of $7 \mathrm{H} 9$ top agar $\left(0.35 \%\right.$ agar w/v), supplemented with $1 \mathrm{mM}$ of $\mathrm{CaCl}_{2}$, and poured onto $7 \mathrm{H} 11$ top agar plates. These plates were incubated at RT for 2 days and an additionallday at $28^{\circ} \mathrm{C}$ before scoring for plaques. Individual plaques were picked and purified through 3 repeated rounds of infection of $M$. smegmatis.

Working phage lyates were prepared by selecting a plate from the final plaque purification process that contained enough phages to clear the entire petri plate. Phage buffer was added to this plate and allowed to stand for $3 \mathrm{~h}$ at RT. Liquid was collected and filtered through $0.2-\mu \mathrm{m}$ syringe filters to produce sterile stocks of purified phages. Phage titer counts were determined by serial dilutions, and lysate stocks were equilibrated to a final concentration of $1 \times 10^{7}$ plaque-forming units (PFUs)/ml.

\subsection{Screening Phages for Biofilm Inhibition}

Phage stocks were individually screened for their abilities to destroy existing M. smegmatis biofilms on MBEC pegs. Two-day-old biofilms of $M$. smegmatis on pegs were submerged into individual wells of 96-well polystyrene plates containing $7 \mathrm{H} 9$ broth containing $1 \mathrm{mM} \mathrm{CaCl}_{2}$ and varying strains of phages. After a 3-day exposure to the phages, CV staining was used to quantify remaining biofilms. The data was expressed as a mean percen- 
tage of the biofilm growth on non-phage treated controls and represents three independent experiments.

\subsection{Prevention of Biofilm Formation}

MBEC assay plates were simultaneously incubated with a combination of planktonic $M$. smegmatis and phages before staining with $\mathrm{CV}$ to determine if phages reduce attachment of bacteria to surfaces. Each peg was exposed to $M$. smegmatis $\left(5 \times 10^{6} \mathrm{CFUs} / \mathrm{ml}\right)$ with increasing concentrations of phages from $1 \times 10^{2}-1 \times 10^{6} \mathrm{PFUs} / \mathrm{ml}$. MBEC plates lowered into the wells were allowed to incubate at RT with gentle shaking $(130 \mathrm{rpm})$ for 4 days before assaying biofilm formation by CV staining. The data was expressed as a mean percentage of the biofilm growth observed on non-phage treated controls. Three independent experiments were performed with 12 replicates for each treatment, producing a total $n=36$.

\subsection{Phage and Antibiotic Biofilm Challenge}

Disruption of existing 2-day-old biofilms was measuredin the presence of the antibiotic isoniazid (INH), bacteriophages, or a combination of INH and phages. MBEC plates with biofilms were submerged into wells of a 96-well plate containing either INH $(10 \mu \mathrm{g} / \mathrm{ml})$ and/or phages $\left(1 \times 10^{3} \mathrm{PFUs} / \mathrm{ml}\right)$ for durations of 24,48 , or $72 \mathrm{~h}$. Two pegs for each treatment were removed, water-bath sonicated, and serially diluted to quantify surviving bacteria.

\subsection{Phage Effects on Dispersal of Biofilm Bells by Mechanical Forces}

Two-day-old biofilms on MBEC plates were exposed to phages for 3 days before the MBEC plates were cut in half. One half of the plate was rinsed by gentle submersion in PBS prior to CV staining and served as a control treatment. The other half was subjected to either sonication or to flowing water prior to CV staining to examine how phage treatment affects subsequent mechanical disruption of biofilms. For the sonication assay, the MBEC plate was placed in microtiter plates containing 7H9 and sonicated for 5 min using a water bath sonicator. Sonication for this length of time caused no change in the viability of $M$. smegmatis cells (data not shown). Data was expressed as a percentage of the CV staining on non-sonicated control pegs of the corresponding treatment.

The effect of running water on phage treated biofilms was examined by anchoring the MBEC plate with the pegs facing upward in a $4 \times 8 \mathrm{~cm}$ trough. The trough was then flooded with deionized water before 25 liters of deionized water was dispensed from a carboy at an average flow of $8.33 \mathrm{~L} / \mathrm{min}$ to ensure consistency between trials (Figure 2). Data was expressed as a percentage of the absorbance of non-water exposed control pegs of the corresponding treatment. Two independent experiments were performed with 12 replicates of each treatment, for a total $\mathrm{n}=24$ for in both sonication and water exposure assays.

\subsection{Effects of Biofilm Age on Phage Mediated Disruption}

Biofilms were grown using the MBEC system for 2 days or 6 days prior to infection. These two durations represent actively growing and steady-state biofilms, respectively (Figure 1). Biofilms on pegs were subjected to destruction by phages for 1 - 3 days as described above, and the effects of phage disrupting biofilms were determined by CV staining. Data was expressed as a percentage of the biofilm growth on the corresponding 2 or 6 day non-phage controls. Two independent experiments were performed with 24 replicates of each treatment for a total $n=48$.

\subsection{Scanning Electron Microscopy}

Pegs containing existing biofilms with and without phage treatment were aseptically removed and fixed in 5\% (v/v) gluteraldehyde/0.1 M cacodylate buffer ( $\mathrm{pH} 7.2$ ) for $24 \mathrm{~h}$ at $4^{\circ} \mathrm{C}$. Pegs were then air dried for 7 days and sputter-coated with gold-palladium prior to being visualized by using a JEOL JSM-6490LV scanning electron microscope, as previously described [38].

\section{Results}

\subsection{Formation of M. smegmatis Biofilms Using MBEC Assay}

The MBEC assay system (also known as the Calgary Biofilm Device) is a high-throughput device for testing 


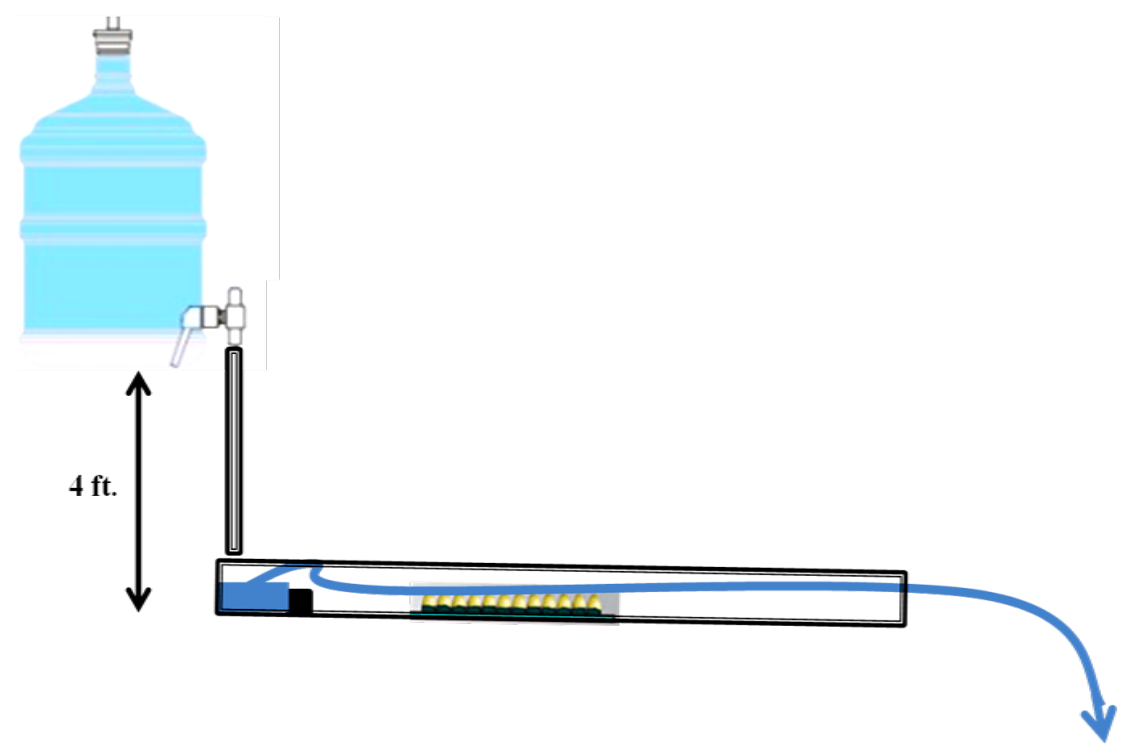

Figure 2. The experimental apparatus used for exposing $M$. smegmatis biofilms to the mechanical force of running water. MBEC top plates were anchored pegs facing up in the trough. A set volume of $25 \mathrm{~L}$ was used to flood the trough at an average flow of 8.33 L/min. Biofilms were then quantified using CV staining.

susceptibility of bacterial biofilms to antimicrobial substances [12] [13]. It consists of a plastic plate with 96 pegs that can be individually submerged into solutions in 96-well tissue culture plates. This assay system has previously been used to study mycobacterial biofilm formation and corresponding resistance to biocides [12] [13] [18]. We first determined if MBEC plates can be used to generate M. smegmatis biofilms that can then be exposed to antibiotics or mycobacteriophages. To determine the optimal time for phage treatment of biofilms, a growth curve for M. smegmatis biofilms was constructed. Biofilm exhibited a sigmoidal growth curve with a 2-log increase during the first 2 days and maximum growth of $9 \times 10^{7} \mathrm{CFU} /$ peg occurring on day 4 (Figure 3). Similar results were obtained from crystal violet (CV) staining (Figure 3). To determine if the sonication of bacterial biofilms was detrimental to M. smegmatis survival, aliquots of planktonic M. smegmatis were sonicated in a water bath and surviving cells enumerated using dilution plate counting. No decrease in viable bacteria was observed during 5 min of sonication (data not shown). Based upon the observed growth of M. smegmatis on MBEC pegs, 2 and 6-day-old biofilms were selected for future phage treatments since these two time points represent actively growing and steady-state biofilms, respectively. This experiment also establishes the validity of using CV staining to quantify biofilms, as the CV stain data closely correlated with the number of viable cells released from biofilms by sonication (Figure 1 ).

\subsection{Screening Phages for Biofilm Inhibition}

Because sphagnum peat bogs are rich sources of environmental mycobacteria [39], these locations were utilized to obtain novel strains of mycobacteriophages. Isolation of environmental mycobacteriophages from a single bog in northern Minnesota resulted in over 40 different purified isolates, which produced clear plaques on $M$. smegmatis lawns that ranged in diameter from $1-4 \mathrm{~mm}$. Thirty-eight phage isolates were screened for their ability to disperse 2-day old biofilms on MBEG pegs, and these isolates exhibited varying abilities to destroy biofilms was monitored by CV staining (Figure 3). The greatest destruction of M. smegmatis biofilms occurred with mycobacteriophage isolates CU 14A, CU 9, DL6, DL 12, and DL 16A that all resulted in CV staining that was $17 \%-19 \%$ the level of non-phage treated biofilms. Isolates DL 9 and DL 11B were lytic phage that resulted in the smallest reductions of biofilm that was $67 \%$ the intensity of non-phage treated biofilms. The remaining 32 phage isolates destroyed existing biofilms in an intermediate range (Figure 3). The three phage isolates CU 14A, DL 2A, and DL 9 (Figure 3, arrowheads) represented strong, intermediate, and weak abilities to destroy biofilms, respectively, and they were chosen for further characterization of their interactions with $M$. smegmatis biofilms. 


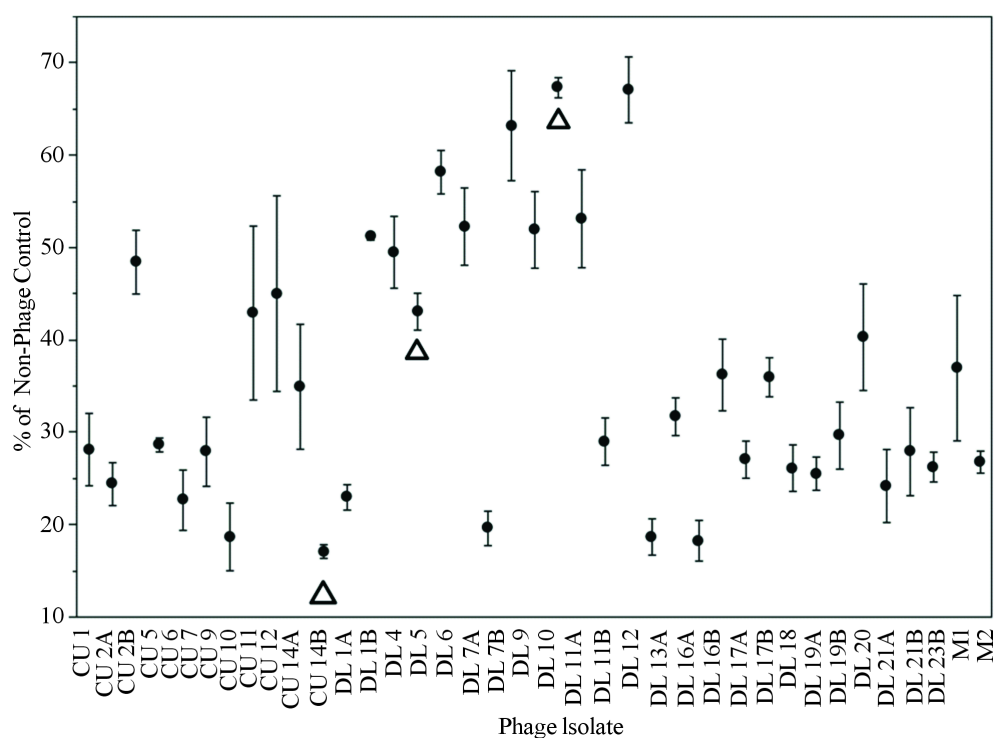

Figure 3. Screening of purified mycobacteriophage isolates for destruction of M. smegmatis biofilms. Data was obtained by CV staining. The bars represent $\mathrm{SE}$ and the points represent staining as a mean percentage of biofilm growth on non-phage treated controls for three independent experiments $(n=3)$. The arrowheads indicate the relative destructive capabilities of the three phage species chosen for further analysis.

\subsection{Prevention of Biofilm Formation}

While mycobacteriophages can be shown to destroy existing M. smegmatis biofilms (Figure 3), we wished to determine if they could also prevent the initial formation of these biofilms. MBEC assay plates were incubated in the presence of a combination of planktonic $M$. smegmatis and phages to determine if bacterial attachment and subsequent biofilm formation would be prevented. Biofilms grown individually in the presence of all three phage species tested showed a decrease in amounts relative to biofilms formed in the absence of phages (Figure 4). The greatest inhibition of biofilm formation (96\% reduction compared to non-phage control pegs) occurred with phage DL 9 using a titer of $1 \times 10^{6} \mathrm{PFU} /$ well. Prevention of biofilm formation directly correlated with increasing phage titers for DL 2A and DL 9, but phage CU 14A showed equal inhibition of biofilms regardless of concentrations used (Figure 4).

\subsection{Phage Effects on Mechanical Biofilm Dispersal}

Based upon CV staining of phage-treated biofilms, phages alone are not sufficient to completely eliminate $M$. smegmatis biofilms (Figure 3 and Figure 4). However, it is possible that in addition to lysing M. smegmatis cells existing in a biofilm that the phages can also disrupt the structures of biofilms and make them more susceptible to dispersion by mechanical disruption. To test this idea, 2-day-old biofilms were treated with phages for 3 days prior to exposing them to disruption by either sonication or flowing water. Following phage treatment and mechanical disruption, pegs were stained with CV and amounts of retained stain were quantified (Figure 5). Sonication produced a $17 \%$ reduction in mass of biofilms that had not been treated with phages (Figure 5(a), black bar). However, sonication of biofilms pretreated with phage CU 14A or DL 2A resulted in 54\% and $40 \%$ reduction in biofilms, respectively, compared to no phage treatment (Figure 5(a)). Phage isolate DL 9 did not elicit significantly greater sonication dispersal compared to non-phage controls. CV staining of the fluid from sonicated biofilms that had been pre-treated with CU 14A and DL 2A showed detached multicellular aggregates in the fluid (data not shown). Such cell aggregates were not seen in fluid from sonicated biofilms that had not been treated with phages. This shows that some phage species have the ability to loosen the matrixes of $M$. smegmatis biofilms.

Instead of sonication, an experiment was performed to measure biofilm dispersal by flowing water following phage treatment. Flowing water is a more natural condition that mycobacterial biofilms encounter in municipal 


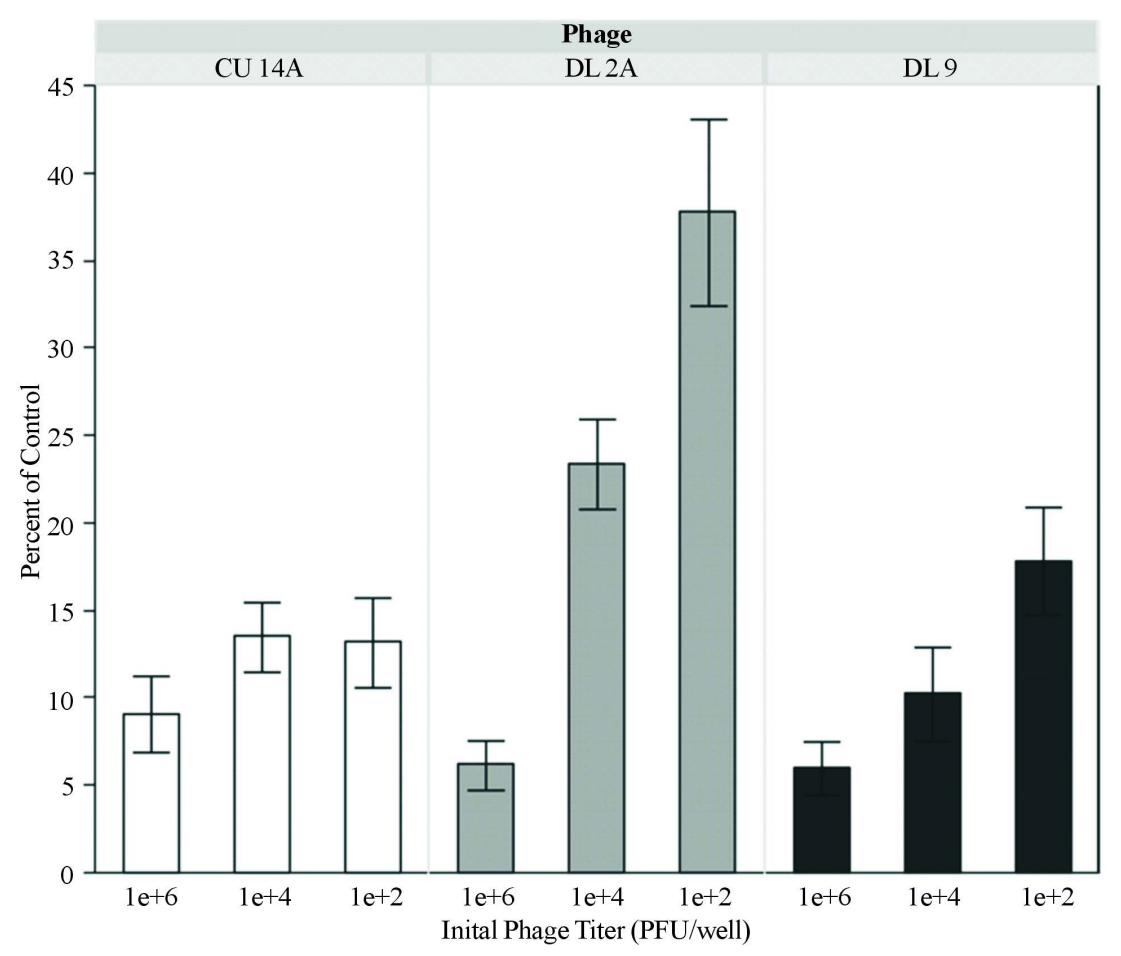

Figure 4. Presence of phage prevents $M$. smegmatis biofilm formation. MBEC plates were incubated 4 days in the presence of $M$. smegmatis and of $10^{2}, 10^{4}$ or $10^{6} \mathrm{PFU} /$ well of phage before quantifying biofilm formation by $\mathrm{CV}$ staining. Bars represent staining as a mean percentage of non-phage control biofilms. Error bars represent the SE of three independent experiments with 12 replicates $(n=36)$.

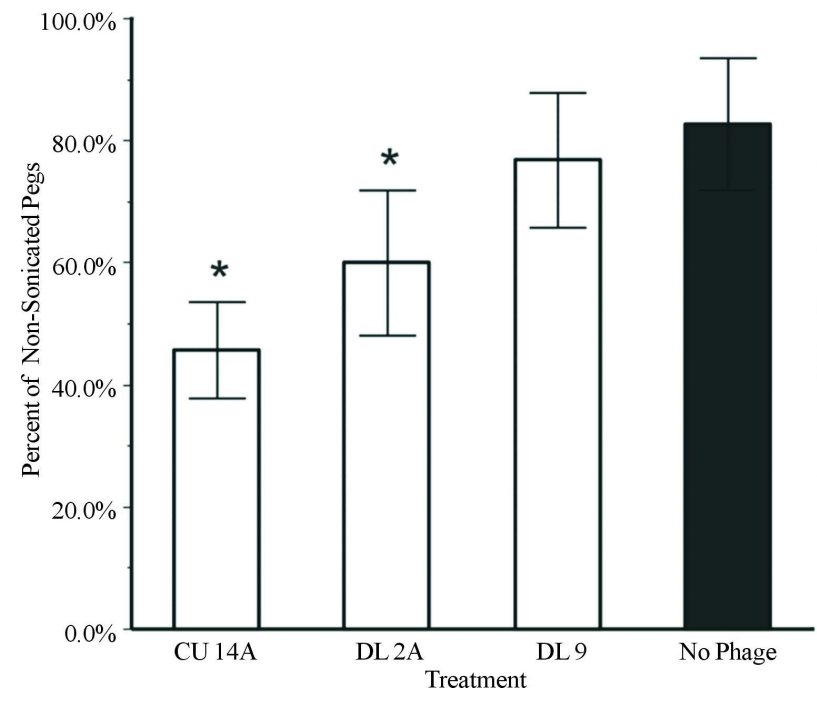

(a)

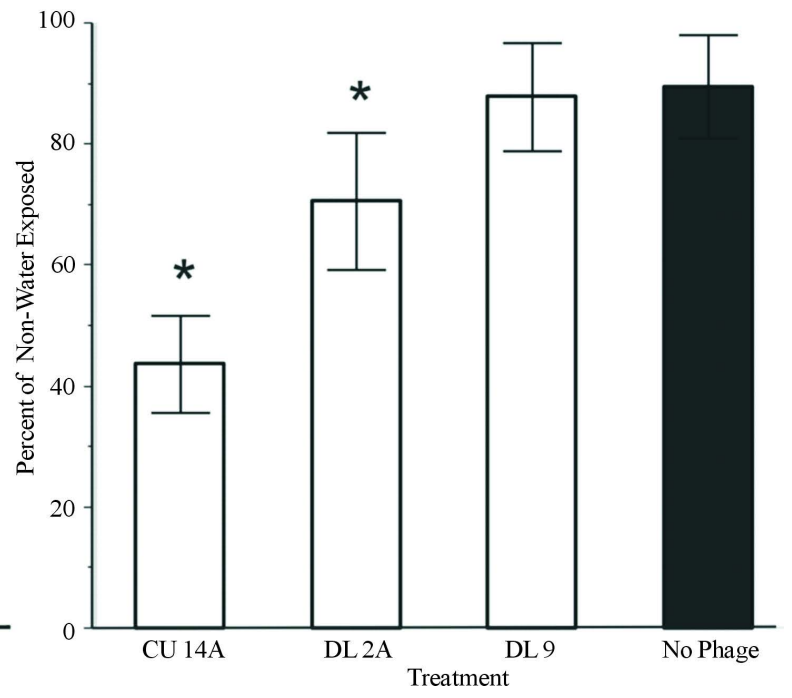

(b)

Figure 5. Phage treatment of biofilms increases M. smegmatis dispersal by mechanical disruption. (a) Dispersal of biofilms in response to sonication. Attached bacteria were quantified using CV staining and data expressed as a percentage of staining on non-sonicated pegs for the corresponding treatment. Error bars represent SE of two independent experiment with 12 replicates $(\mathrm{n}=48)$. *Significant reduction in biomass compared to the non-phage treatment (Tukey's HSD test, $\mathrm{P}<0.001)$. (b) Dispersal of biofilms in response to flowing water. Attached bacteria were quantified using CV staining and data expressed as a percentage of staining on non-water exposed pegs for the corresponding treatment. Error bars represent SE of two independent experiment with 12 replicates $(\mathrm{n}=48)$. *Significant reduction in biomass compared to the non-phage treatment (Tukey’s HSD test, $\mathrm{P}<0.001$ ). 
water sources. Exposure of biofilms to phages CU 14A and DL 2A produced a greater reduction in biomass (51\% and $30 \%$, respectively) compared to only a $11 \%$ reduction of biofilms by water flow if the biofilms were not exposed to phages $(\mathrm{P}<0.001$, Figure 5(b)). Exposure to phage DL 9 resulted in no significant increase in water dispersal compared to non-phage controls.

\subsection{Effects of Biofilm Age on Phage-Mediated Dispersal}

Initial screening of mycobacteriphages was performed using actively developing M. smegamatis biofilms with a single exposure length to phages (Figure 3). Biofilms will exhibit multiple phenotypes over the course of development, including differential regulation of genes and expression of proteins [40]. We tested if different stages of biofilm development had different susceptibilities to phage-mediated dispersal. To determine the effect of biofilm maturation on phage susceptibility, biofilms were grown for either 2 or 6 days prior to infection. Two and 6 days produce actively growing biofilms and steady state biofilms, respectively (Figure 1). For phage species CU 14A and DL 2A, the greatest biofilm reduction was seen for 2-day-old biofilms instead of for the more mature 6 day-old biofilms $(\mathrm{P}<0.05$, Figure 6$)$.

Scanning electron microscopy (SEM) was used to directly visualize the effect of phages on M. smegmatis biofilms on the MBEC pegs (Figure 7). Greater cell confluence can be observed in non-phage controls (Figure 7(a) and Figure 7(c)) compared to phage-treated pegs (Figure 7(b) and Figure 7(d)). Extensive EPS can be seen in the 6-day-old biofilms showing a uniform mat of cells (Figure 7(c)). The 2-day-old biofilm showed EPS production, but the biofilm structure is much more irregular than the 6-day-old biofilm and contains fluid-filled voids (compare Figure 7(a) with Figure 7(c)). Therefore, SEM analysis confirms that the age of M. smegmatis biofilms matters with regards to surface area that is potentially exposed to attack by lytic phages.

\section{Discussion}

Recent studies have shown bacteriophages can disrupt biofilms of a variety of different bacteria in which the extracellular matrixes are typically polysaccharide in nature [41]-[46]. However, it was previously unknown if phages could disrupt mycobacterial biofilms with lipid-based extracellular matrixes [47]. Here we show myco-

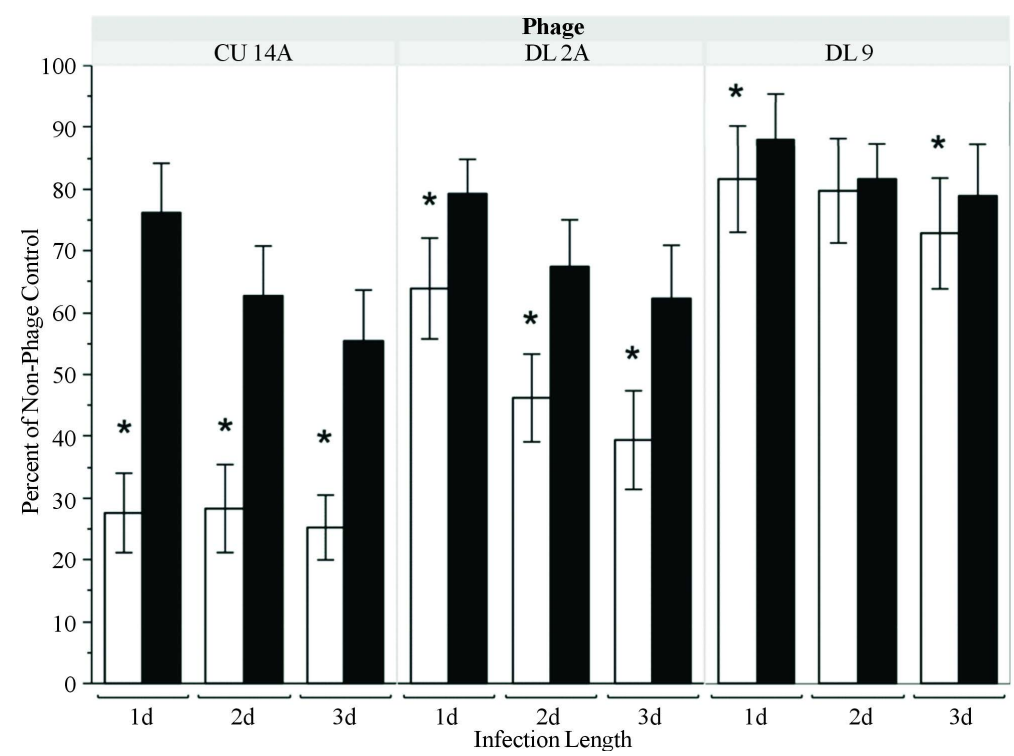

Figure 6. Effects of biofilm age on biomass reduction. MBEC devices were incubated either two or six days prior to infection, and biofilms were measured using CV staining. White bars represent staining as mean percentage of biofilm growth on non-phage treated controls for 2-day-old biofilms and black bars represent the mean percentage growth on non-phage treated 6-day-old biofilms. Error bars represent the standard deviation of two independent experiments with 24 replicates $(n=48)$. *Significantly greater reduction in biomass compared to 6 day biofilms (light bands; Tukey's HSD test, $\mathrm{P}<0.05)$. 

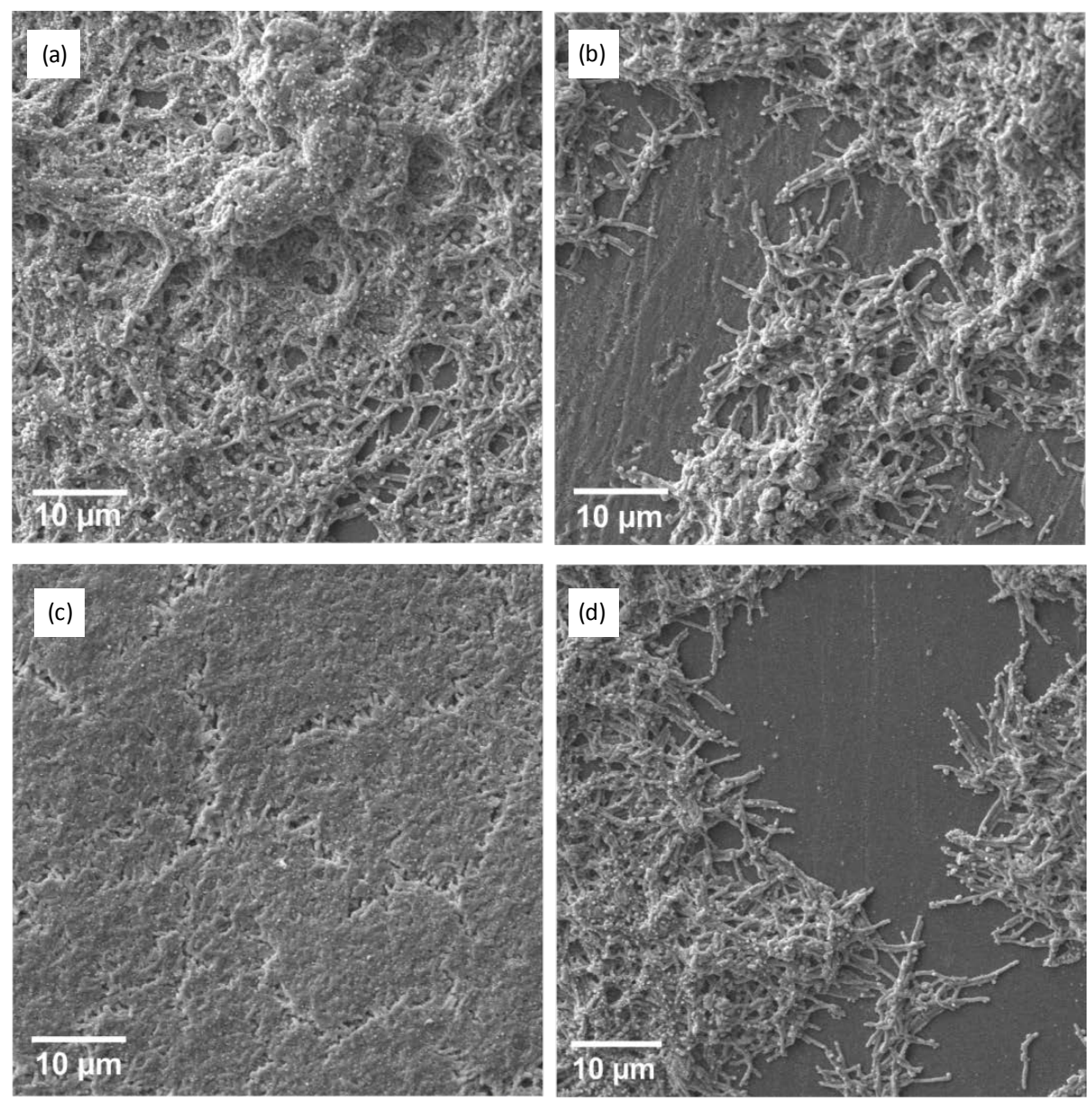

Figure 7. SEM of $M$. smegmatis (50,000× magnification) growing on an MBEC device after 2-day incubation and no phage treatment (a), 2-day incubation plus 3-day infection with CU 14A (b), 6-day incubation and no phage treatment (c), and 6-day incubation and 3-day infection with CU $14 \mathrm{~A}(\mathrm{~d})$.

bacteriophages isolated from a peat bog in northern Minnesota have a wide diversity of abilities to damage existing $M$. smegmatis biofilms. Bogs are known sites of mycobacterial diversity, and it is likely that phages and their host species have complex ecological relationships in these environments. The wide variety of biocidal activity of these phages on M. smegmatis biofilms (Figure 3) parallels the enormous genetic diversity found in different mycobacteriophages [48] [49]. Of the 39 mycobacteriophage species tested for biocidal activity on $M$. smegmatis, all exhibited at least some destructive potential on existing biofilms ranging from strong to intermediate to weak disruption activity as exemplified by the three phage isolates CU 14A, DL 2A, and DL 9, respectively. All three of these phage species produced clear plagues on M. smegmatis in soft agar (data not shown), but these three phage isolates had marked differences in their abilities to reduce existing biofilms (Figure 3, arrowheads). In addition to disrupting existing biofilms, these three mycobacteriophage species can also inhibit the initial formation of biofilms (Figure 4). Phage variables that may affect the varying amounts of biofilm disruption include phage size (smaller phages might penetrate matrixes of biofilms more easily), burst size (more phages might mean greater disruption of host cells), charge of phage particles (the matrixes of mycobacterial biofilms are believed to be hydrophobic) [47], host cell receptors (accessibilities to different receptors in biofilms), and phage genes (certain phages may have enzymes that disrupt biofilms differently).

While no phage species tested here could eliminate all attached M. smegmatis from MBEC pegs (Figure 3), at least two species showed an ability to disrupt biofilms and set them up for further disruption by the mechanical forces of sonication and flowing water (Figure 5). These results are relevant both to biofilm control strategies and to better understanding mycobacterial ecology. Efforts to control biofilms by mechanical measures could be 
enhanced in the presence of phages that make cells easier to dislodge. When biofilms mature, they are capable of releasing planktonic cells into the environment to colonize new areas. Loosening of mycobacteria from biofilms by phages could enhance dispersal of live bacteria. Therefore, in addition to lysing bacteria it is possible that mycobacteriophages in nature help accelerate the spread of mycobacteria from stationary biofilms into the wider environment.

\section{Conclusion}

This study shows the potential for phages to prevent and to disrupt mycobacteria contamination on surfaces. Future studies will focus on the molecular characterization of phages, like CU 14A, which are highly effective in reducing biofilms. The approaches described here could also be applied to isolating phages to disrupt environmental biofilms of known human pathogens like M. marinum and M. ulcerans [18] [19].

\section{References}

[1] Tortoli, E. (2003) Impact of Genotypic Studies on Mycobacterial Taxonomy: The New Mycobacteria of the 1990s. Clinical Microbiology Reviews, 16, 319-354. http://dx.doi.org/10.1128/CMR.16.2.319-354.2003

[2] Colville, A. (1993) Retrospective Review of Culture-Positive Mycobacterial Lymphadenitis Cases in Children in Nottingham, 1979-1990. European Journal of Clinical Microbiology \& Infectious Diseases 12, 192-195. http://dx.doi.org/10.1007/BF01967110

[3] De Groote, M.A. and Huitt, G. (2006) Infections Due to Rapidly Growing Mycobacteria. Clinical Infectious Diseases, 42, 1756-1763. http://dx.doi.org/10.1007/BF01967110

[4] Han, X.Y., Dé, I. and Jacobson, K.L. (2007) Rapidly Growing Mycobacteria Clinical and Microbiologic Studies of 115 Cases. American Journal of Clinical Pathology, 128, 612-621. http://dx.doi.org/10.1309/1KB2GKYT1BUEYLB5

[5] Primm, T.P., Lucero, C.A. and Falkinham, J.O. (2004) Health Impacts of Environmental Mycobacteria. Clinical Microbiology Reviews, 17, 98-106. http://dx.doi.org/10.1128/CMR.17.1.98-106.2004

[6] Falkinham $3^{\text {rd }}$ J.O. (1996) Epidemiology of Infection by Nontuberculous Mycobacteria. Clinical Microbiology Reviews, 9, 177-215.

[7] Stoodley, P., Sauer, K., Davies, D. and Costerton, J.W. (2002) Biofilms as Complex Differentiated Communities. Annual Review of Microbiology, 56, 187-209. http://dx.doi.org/10.1146/annurev.micro.56.012302.160705

[8] Costerton, J., LewandowskiI, Z., Caldwell, D., Korber, D. and Lappinscott, H. (1995) Microbial Biofilms. Annual Review of Microbiology, 49, 711-745. http://dx.doi.org/10.1146/annurev.mi.49.100195.003431

[9] Lasa, I. (2006) Towards the Identification of the Common Features of Bacterial Biofilm Development. International Journal of Food Microbiology, 9, 21-28.

[10] Ojha, A.K., Trivelli, X., Guerardel, Y., Kremer, L. and Hatfull, G.F. (2010) Enzymatic Hydrolysis of Trehalosedimycolate Releases Free Mycolic Acids during Mycobacterial Growth in Biofilms. Journal of Biological Chemistry, 285, 17380-17389. http://dx.doi.org/10.1074/jbc.M110.112813

[11] Whitchurch, C.B., Tolker-Nielsen, T., Ragas, P.C. and Mattick, J.S. (2002) Extracellular DNA Required for Bacterial Biofilm Formation. Science, 295, 1487-1487. http://dx.doi.org/10.1126/science.295.5559.1487

[12] Bardouniotis, E., Ceri, H. and Olson, M.E. (2003) Biofilm Formation and Biocide Susceptibility Testing of Mycobacterium fortuitum and Mycobacterium marinum. Current Microbiology, 46, 28-32. http://dx.doi.org/10.1007/s00284-002-3796-4

[13] Bardouniotis, E., Huddleston, W., Ceri, H. and Olson, M.E. (2001) Characterization of Biofilm Growth and Biocide Susceptibility Testing of Mycobacterium phlei Using the MBEC ${ }^{\text {TM }}$ Assay System. FEMS Microbiology Letters, 203, 263-267. http://dx.doi.org/10.1016/s0378-1097(01)00364-0

[14] Hall-Stoodley, L. and Lappin-Scott, H. (1998) Biofilm Formation by the Rapidly Growing Mycobacterial Species $M y$ cobacterium fortuitum. FEMS Microbiology Letters, 168, 77-84. http://dx.doi.org/10.1111/j.1574-6968.1998.tb13258.x

[15] Schwartz, T., Kalmbach, S., Hoffmann, S., Szewzyk, U. and Obst, U. (1998) PCR-Based Detection of Mycobacteria in Biofilms from a Drinking Water Distribution System. Journal of Microbiological Methods, 34, 113-123. http://dx.doi.org/10.1016/S0167-7012(98)00081-5

[16] September, S., Brözel, V. and Venter, S. (2004) Diversity of Nontuberculoid Mycobacterium Species in Biofilms of Urban and Semiurban Drinking Water Distribution Systems. Applied and Environmental Microbiology, 70, 7571-7573. http://dx.doi.org/10.1128/AEM.70.12.7571-7573.2004

[17] Adetunji, V.O., Kehiude, A.O., Bolatito, O.K. and Chen, J. (2014) Biofilm Formation by Mycobacterium bois: Influence of Surface Kind and Temperatures of Sanitizer Treatments on Biofilm Control. BioMed Research International, 
2014, Article ID: 210165. http://dx.doi.org/10.1155/2014/210165

[18] Hall-Stoodley, L., Brun, O.S., Polshyna, G. and Barker, L.P. (2006) Mycobacterium marinum Biofilm Formation Reveals Cording Morphology. FEMS Microbiology Letters, 257, 43-49. http://dx.doi.org/10.1111/j.1574-6968.2006.00143.x

[19] Marsollier, L., Brodin, P., Jackson, M., Kordulakova, J., Tafelmeyer, P., Carbonnelle, E., et al. (2007) Impact of Mycobacterium ulcerans Biofilm on Transmissibility to Ecological Niches and Buruli Ulcer Pathogenesis. PLoS Pathogens, 3, e62. http://dx.doi.org/10.1371/journal.ppat.0030062

[20] Falkinham III, J., Iseman, M., Haas, P. and Soolingen, D. (2008) Mycobacterium avium in a Shower Linked to Pulmonary Disease. Journal of Water and Health, 6, 209-213.

[21] Feazel, L.M., Baumgartner, L.K., Peterson, K.L., Frank, D.N., Harris, J.K. and Pace, N.R. (2009) Opportunistic Pathogens Enriched in Showerhead Biofilms. Proceedings of the National Academy of Sciences of the United States of America, 106, 16393-16399. http://dx.doi.org/10.1073/pnas.0908446106

[22] Nishiuchi, Y., Maekura, R., Kitada, S., Tamaru, A., Taguri, T., Kira, Y., et al. (2007) The Recovery of Mycobacterium avium-intracellulare Complex (MAC) from the Residential Bathrooms of Patients with Pulmonary MAC. Clinical Infectious Diseases, 45, 347-351. http://dx.doi.org/10.1086/519383

[23] Espeland, E. and Wetzel, R. (2001) Complexation, Stabilization, and UV Photolysis of Extracellular and SurfaceBound Glucosidase and Alkaline Phosphatase: Implications for Biofilm Microbiota. Microbial Ecology, 42, 572-585. http://dx.doi.org/10.1007/s00248-001-1023-7

[24] Le Magrex-Debar, E., Lemoine, J., Gellé, M., Jacquelin, L. and Choisy, C. (2000) Evaluation of Biohazards in Dehydrated Biofilms on Foodstuff Packaging. International Journal of Food Microbiology, 55, 239-243. http://dx.doi.org/10.1016/S0168-1605(00)00177-X

[25] McNeill, K. and Hamilton, I. (2003) Acid Tolerance Response of Biofilm Cells of Streptococcus mutans. FEMS Microbiology Letters, 221, 25-30. http://dx.doi.org/10.1016/S0378-1097(03)00164-2

[26] Donlan, R.M. and Costerton, J.W. (2002) Biofilms: Survival Mechanisms of Clinically Relevant Microorganisms. Clinical Microbiology Reviews, 15, 167-193. http://dx.doi.org/10.1128/CMR.15.2.167-193.2002

[27] Tengra, F.K., Dahl, J.L., Dutton, D., Caberoy, N.B., Coyne, L. and Garza, A.G. (2006) CbgA, a Protein Involved in Cortex Formation and Stress Resistance in Myxococcus xanthus Spores. Journal of Bacteriology, 188, 8299-8302. http://dx.doi.org/10.1128/JB.00578-06

[28] Covert, T.C., Rodgers, M.R., Reyes, A.L. and Stelma, G.N. (1999) Occurrence of Nontuberculous Mycobacteria in Environmental Samples. Applied and Environmental Microbiology, 65, 2492-2496.

[29] Gomez-Smith, C.K., La Para, T.M. and Hozalski, R.M. (2015) Sulfate Reducing Bacteria and Mycobacteria Dominate the Biofilm Communities in a Chloraminated Drinking Water Distribution System. Environmental Science \& Technology, 49, 8432-8440. http://dx.doi.org/10.1021/acs.est.5b00555

[30] Le Dantec, C., Duguet, J., Montiel, A., Dumoutier, N., Dubrou, S. and Vincent, V. (2002) Occurrence of Mycobacteria in Water Treatment Lines and in Water Distribution Systems. Applied and Environmental Microbiology, 68, 53185325. http://dx.doi.org/10.1128/AEM.68.11.5318-5325.2002

[31] Tsintzou, A., Vantarakis, A., Pagonopoulou, O., Athanassiadou, A. and Papapetropoulou, M. (2000) Environmental Mycobacteria in Drinking Water before and after Replacement of the Water Distribution Network. Water, Air, and Soil Pollution, 120, 273-282. http://dx.doi.org/10.1023/A:1005266827726

[32] Cerca, N., Oliveira, R. and Azeredo, J. (2007) Susceptibility of Staphylococcus epidermidis Planktonic Cells and Biofilms to the Lytic Action of Staphylococcus Bacteriophage K. Letters in Applied Microbiology, 45, 313-317. http://dx.doi.org/10.1111/j.1472-765X.2007.02190.x

[33] Chhibber, S., Bansal, S. and Kaur, S. (2015) Disrupting the Mixed Species Biofilm of Klebsiella pneumonia B5055 and Pseudomonas aeruginosa PAO Using Bacteriophages Alone or in Combination with Xylitol. Microbiology, 161, 1369-1377.

[34] Sass, P. and Bierbaum, G. (2007) Lytic Activity of Recombinant Bacteriophage $\varphi 11$ and $\varphi 12$ Endolysinson Whole Cells and Biofilms of Staphylococcus aureus. Applied and Environmental Microbiology, 73, 347-352. http://dx.doi.org/10.1128/AEM.01616-06

[35] Sharma, M., Ryu, J. and Beuchat, L. (2005) Inactivation of Escherichia coli O157:H7 in Biofilm on Stainless Steel by Treatment with an Alkaline Cleaner and a Bacteriophage. Journal of Applied Microbiology, 99, 449-459. http://dx.doi.org/10.1111/j.1365-2672.2005.02659.x

[36] Sillankorva, S., Oliveira, R., Vieira, M. and Azeredo, J. (2008) Real-Time Quantification of Pseudomonas fluorescens Cell Removal from Glass Surfaces Due to Bacteriophage $\varphi S 1$ Application. Journal of Applied Microbiology, 105, 196202. http://dx.doi.org/10.1111/j.1365-2672.2008.03743.x 
[37] Knezevic, P. and Petrovic, O. (2008) A Colorimetric Microtiter Plate Method for Assessment of Phage Effect on Pseudomonas aeruginosa Biofilm. Journal of Microbiological Methods, 74, 114-118. http://dx.doi.org/10.1016/j.mimet.2008.03.005

[38] Teng, R. and Dick, T. (2003) Isoniazid Resistance of Exponentially Growing Mycobacterium smegmatis Biofilm Culture. FEMS Microbiology Letters, 227, 171-174. http://dx.doi.org/10.1016/S0378-1097(03)00584-6

[39] Kazda, J. (2000) The Ecology of Mycobacteria. Klewer Academic Publishers, Dordrecht. http://dx.doi.org/10.1007/978-94-011-4102-4

[40] Sauer, K. (2003) The Genomics and Proteomics of Biofilm Formation. Genome Biology, 4, 219. http://dx.doi.org/10.1186/gb-2003-4-6-219

[41] Carson, L., Gorman, S.P. and Gilmore, B.D. (2010) The Use of Lytic Bacteriophages in the Prevention and Eradication of Biofilms of Proteus mirabilis and Escherichia coli. FEMS Immunology \& Medical Microbiology, 59, 447-455. http://dx.doi.org/10.1111/j.1574-695x.2010.00696.x

[42] Chhibber, S., Nag, D. and Bansal, S. (2013) Inhibiting Biofilm Formation by Klebsiella pneumonia B5055 Using an Iron Antagonizing Molecule and a Bacteriophage. BMC Microbiology, 13, 174.

[43] Chibeu, A., Lingohr, E.J., Masson, L., Manges, A., Harel, J., Ackermann, H.W., Kropinski, A.M. and Boerlin, P. (2012) Bacteriophages with the Ability to Degrade Uropathogenic Escherichia coli Biofilms. Viruses, 4, 471-487.

[44] Kelly, D., McAuliffe, O., Ross, R.P. and Coffey, A. (2012) Prevention of Staphylococcus aureus Biofilm Formation and Reduction in Established Biofilm Density Using a Combination of Phage K and Modified Derivatives. Letters in Applied Microbiology, 54, 286-291.

[45] Siringan, P., Connerton, P.L., Payne, R.J. and Connerton, I.F. (2011) Bacteriophage-Mediated Dispersal of Campylobacter jejuni Biofilms. Applied and Environmental Microbiology, 77, 3320-3326. http://dx.doi.org/10.1128/AEM.02704-10

[46] Zhang, Y., Hunt, H.K. and Hu, Z. (2013) Application of Bacteriophages to Selectively Remove Pseudomonas aeruginosa in Water and Wastewater Filtration Systems. Water Research, 47, 4507-4518.

[47] Zambrano, M.M. and Kolter, R. (2005) Mycobacterial Biofilms: A Greasy Way to Hold It Together. Cell, 123, 762764. http://dx.doi.org/10.1016/j.cell.2005.11.011

[48] Hatfull, G.F. (2010) Mycobacteriophages: Genes and Genomes. Annual Review of Microbiology, 64, 331-356. http://dx.doi.org/10.1146/annurev.micro.112408.134233

[49] Hatfull, G.F., Cresawn, S.G. and Hendrix, R.W. (2008) Comparative Genomics of the Mycobacteriophages: Insights into Bacteriophage Evolution. Research in Microbiology, 159, 332-339. http://dx.doi.org/10.1016/j.resmic.2008.04.008 